\title{
Making Legacy Thermal Storage Heating fit for the Smart Grid
}

\author{
P.J. Boait ${ }^{a^{*}}$, J.R. Snape ${ }^{\text {a }}$, S.J. Darby ${ }^{\text {, }}$,J. Hamilton ${ }^{\text {c }}$, R.J.R. Morris ${ }^{\text {d }}$ \\ ${ }^{a}$ Institute of Energy and Sustainable Development, De Montfort University, Queens Building, \\ The Gateway, Leicester LE1 9BH, UK. Emails: p.boait@dmu.ac.uk, jsnape@dmu.ac.uk \\ ${ }^{\mathrm{b}}$ Environmental Change Institute, School of Geography and the Environment, University of \\ Oxford, South Parks Road, Oxford, OX1 3QY, UK. Email: sarah.darby@ouce.ox.ac.uk \\ ${ }^{c}$ Department of Geography and Environmental Science, School of Archaeology, Geography and \\ Environmental Science, The University of Reading, Whiteknights, PO Box 227,Reading, RG6 6AB, \\ UK. Email: e.j.hamilton@pgr.reading.ac.uk \\ ${ }^{d}$ Energy Local Ltd, 8 Twyford Crescent, LondonW3 9PP. Email: robin@energylocal.co.uk
}

\section{Abstract}

Thermal storage heaters, charged using overnight off-peak electricity, have been used for domestic space heating in the UK and other countries since the 1980s. However, they have always been difficult for consumers to manage efficiently and, with the advent of a high proportion of renewables in the electricity generation mix, the time of day when they are charged needs to be more flexible. There is also a need to reduce peaks in the demand profile to allow distribution networks to support new sources of demand such as electric vehicles. We describe a trial of a smart control system that was retrofitted to a group of six dwellings with this form of heating, with the objectives of providing more convenient and efficient control for the users while varying the times at which charging is performed, to flatten the profile of demand and make use of locally-generated renewable electricity. The trial also employs a commercially-realistic combination of a static time-of-day tariff with a real time tariff dependent on local generation, to provide consumers with the opportunity and incentive to reduce their costs by varying times of use of appliances. Results from operation over the

\footnotetext{
* Corresponding author. Tel.: +44-124-251-1278; fax +44-116-257-7981
} 
2015-16 heating season indicate that the objectives are largely achieved. It is estimated that on an annualised and weather-adjusted basis most of the users have consumed less electricity than before intervention and their costs are less on the trial tariffs. Critical factors for success of this form of system are identified, particularly the need to facilitate hands-on control of heating by thrifty users and the importance of an effective and sustained user engagement programme when introducing the technology, to ensure users gain confidence through a readily-accessible source of support and advice.

Keywords: Thermal storage; demand response; time-of-day tariff; electric heating; smart meter 


\section{Introduction}

In March 2016 the UK's National Infrastructure Commission (NIC) published a report [1] entitled "Smart Power" arguing that investment in interconnection, storage, and demand flexibility "could save consumers up to $£ 8$ billion a year by 2030 , help the UK meet its 2050 carbon targets, and secure the UK's energy supply for generations." These attractive benefits arise from the urgent need faced by many national electricity systems to adapt to an increasing level of intermittent and geographically distributed renewable generation on the supply side, while meeting rising demand from increasing adoption of technologies such as heat pumps and electric vehicles. Similar findings have been documented elsewhere, for example Pudjianto et al. [2] identify a potential value of up to $£ 30 \mathrm{Bn}$ in deferred or avoided network reinforcement costs up to 2050 that can be obtained from demand flexibility that reduces the peak load on local distribution networks. The regulatory changes needed to realise these benefits are now being actively pursued [3].

In this paper we report on a practical trial that realises part of the vision for storage and demand flexibility described in the NIC report. It exploits electrically-charged thermal storage heating (generally known as storage heating) - a form of electric space heating that is found in 1.3 million homes in England [4] and is employed in other temperate countries such as France and New Zealand. Storage heating appliances comprise an insulated enclosure containing high thermal capacity ceramic bricks that are heated by a resistive heating element. The heat energy held in the bricks is then progressively released depending on the setting of vents in the insulating enclosure. Figures 1 and 2 illustrate a typical appliance. Conventionally in the UK these appliances are used in combination with an electricity tariff known as 'Economy 7' which allows them to be charged overnight during a fixed 7 hour interval at a low tariff. The majority of dwellings with this form of heating are rented and relatively small [4] as is the case for those in this trial. This implies that users are more likely 
to suffer from poverty, while fuel poverty, a more specific measure of fuel costs in relation to income, currently arises in $16 \%$ of electrically-heated English households, compared with $11 \%$ for all households [5]. The cost impact is therefore an important factor in the trial.
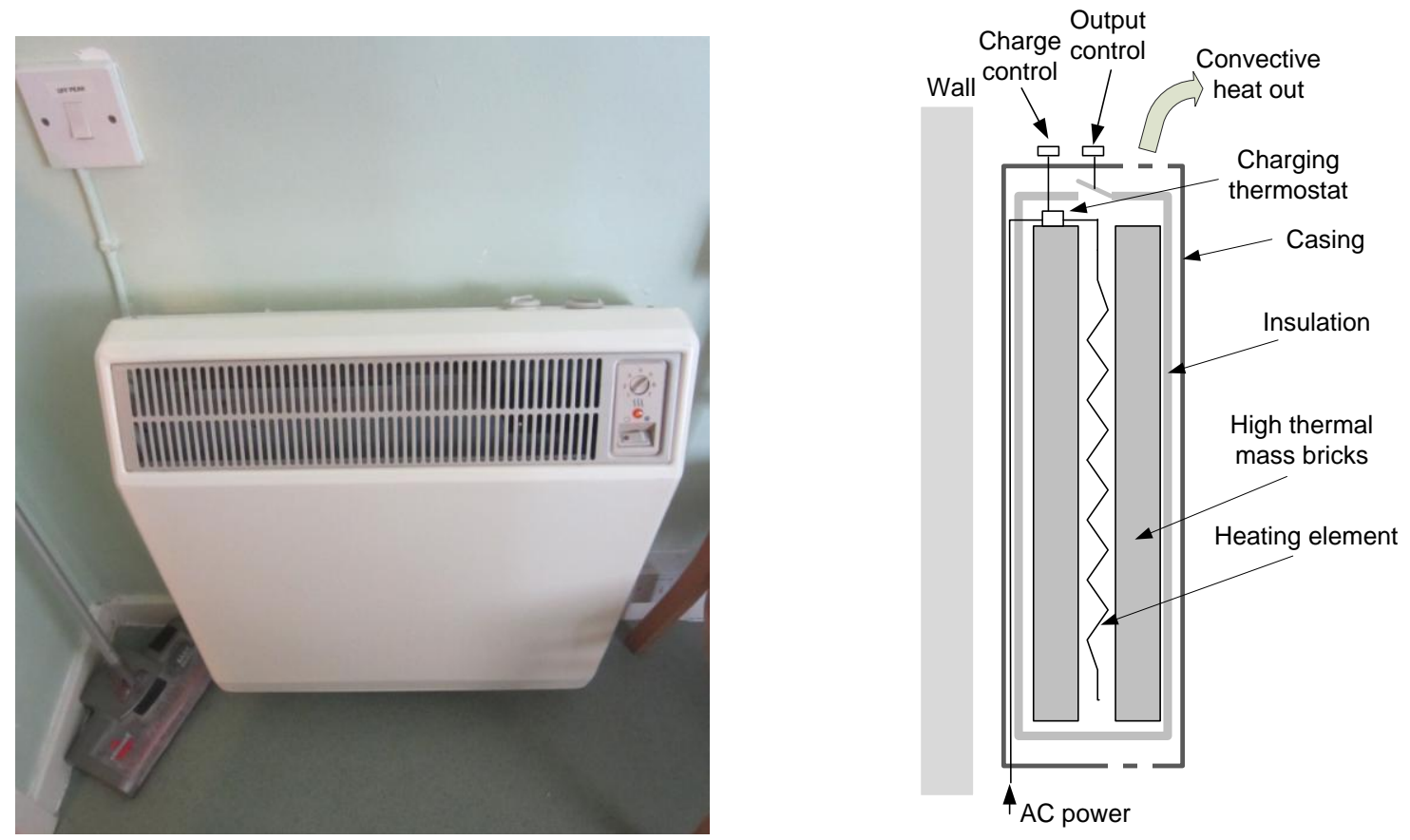

Fig.1 Storage heater

Fig. 2 Storage heater simplified cross-section

There are two major disadvantages in this system of heating as currently employed:

- The appliances are actually quite difficult to use efficiently because energy charge and discharge are regulated by separate manual controls (seen at top right of the unit in Figure 1) whose interaction and weather dependence are hard to comprehend.

- There is no flexibility to vary the time of day when charging is performed to reflect the dynamic availability of electricity supply, particularly from renewable generation. 
A form of "smart" control that can be retrofitted to these legacy appliances ${ }^{1}$ is therefore needed that addresses these weaknesses by providing user-friendly controls and allowing the charging time to be shifted throughout the day in a responsive way that reconciles the comfort needs of the user with grid and generation opportunities and constraints. We describe a control system which provides these features and report results from a trial. The rest of the paper is organised as follows. Section 2 provides more detail on the technical and human factors relating to storage heating. Section 3 describes our control system and the form of prototype trial that has been undertaken, then Section 4 summarises results from the trial. The implications of results are discussed in 5 and conclusions presented in 6.

\section{Background}

2.1 Storage heating

In the UK storage heating tends to be deployed in smaller dwellings such as flats or maisonettes, motivated by the low installation and maintenance cost compared to gas central heating (which also gives rise to more stringent building construction requirements particularly for multi-storey buildings). This results in a typical installation of one large and two or three smaller heaters having a total input power of about $8 \mathrm{~kW}$ and thermal storage capacity of $56 \mathrm{kWh}$. So the $1.3 \mathrm{M}$ households in England have an aggregate capacity in the region of $73 \mathrm{GWh}$. As a resource for grid management it compares favourably with the UK grid's total pumped storage capacity of $30 \mathrm{GWh}[6]$. This potential, combined with the need to avoid the network impact of large numbers of storage heaters switching on simultaneously, was recognised in the 1970s leading to the introduction of the Radio Teleswitch system [7] in 1984. This makes use of the BBC Radio 4 long wave transmission on $198 \mathrm{kHz}$ to carry a

\footnotetext{
${ }^{1}$ A trial of new-generation, smartly-controlled storage heaters is under way in Ireland, German and Latvia, in the EC-funded project RealValue. The project runs from 2015-18.
} 
very low data rate signal to receivers linked to 'Economy 7' meters that record consumption on a two-band tariff that provides for cheap electricity during seven night-time hours. The signal causes the meter to adjust the time window over which the connected storage heaters are charged. However, the time variations are limited to the overnight period and constrained to 256 options by the signal format, while the introduction of an electricity market enabling consumers to switch suppliers easily has caused the contractual basis for operation of this functionality to be lost for many installations. By 2013 the regulator Ofgem found that only 550,000 Teleswitch installations were in use and some associated tariffs were poor value [8]. Another similar system called CycloControl, providing weather-dependent control of storage heaters for flats in London, was abandoned by the service provider in 2015 [9].

A more modern system of demand management is clearly needed. The introduction of half-hour metering under the UK smart meter programme [10] will allow validation of any demand response mechanism from metering data so that consumers can be rewarded for participation. Since heating homes with low carbon electricity is a major element in UK strategy for reducing carbon emissions [11] and the value to the electricity system of electric heating that can deliver demand response is likely to increase by 2030 to between $£ 0.015$ and $£ 0.03$ per $\mathrm{kWh}$ of renewable energy consumed [12] it is realistic to expect that this form of heating, which is cheap to install and very reliable, will become more economically attractive. This potential for sustained and possibly increased use applies throughout northern Europe [13], [14], [15].

However, to realise this potential the legacy user interface to storage heaters is in need of modernisation. It comprises an input (charging) control, which is essentially a thermostat limiting the temperature of the storage bricks, and an output control that regulates the release of heat through circulation of air round the bricks. For efficient operation the input control 
needs to be set late in the day, to obtain the right amount of heat overnight given the weather expected the next day. The output control has to be set to a minimum overnight to reduce losses during charging and then progressively opened during the day to maintain comfort. While some users have the patience and foresight to execute this procedure successfully, others struggle.

Consequently many energy advice agencies and social housing organisations provide websites and videos explaining the use of these controls [16], [17]. But the absence of any direct relationship between control calibration and the room temperature outcome requires (even for users who understand the principles) considerable experience gained by trial and error for satisfactory results. The difficulties in controlling timing and intensity of storage heating are probably the single most important factor in explaining its relative unpopularity [18], [19]. This need for more intuitive and effective controls is a common theme from research into domestic heating [20], [21].

The ability to operate time-of-day dependent tariff structures is usually given as one of the main reasons for the introduction of smart meters in the UK and elsewhere. However, a basic difficulty arises if an automated domestic appliance (such as a storage heater under "smart" control) is given the objective of minimising the cost incurred under a time-of-day tariff while meeting the consumer's heating (or other) needs. There is a strong tendency for a population of such devices to 'choose' the same combination of time and tariff resulting in a peak in aggregate demand which is unhelpful for both electricity suppliers and distribution network operators [22], [23]. If the tariff is closely coupled to wholesale electricity pricing this effect could cause instability in the entire electricity system [24]. This issue must be resolved to deliver the smart grid vision described in [1]. 
Two of the present authors (Boait and Snape) have sought to investigate this problem using agent-based modelling techniques to simulate the electricity system. This modelling project called CASCADE [25] included both weather and behaviour driven components of electricity demand such as electric heating and wet appliances. The outcome was an approach to automated domestic demand response called "demand shaping" [26] with the following features:

- The signal sent by electricity suppliers to a consumer's smart appliance is a daily 48 -value vector $S$ that is not inherently a tariff, but structured so that high values deter, and low values attract, electricity use in each half hour timeslot of the next 24 hours. The length of 48 is employed because the electricity market conventionally operates in half-hour timeslots.

- Automated appliance controls respond to this signal by scheduling demand within a time window that meets user needs but with a probability in proportion to the attractiveness of the signal in each timeslot. The user needs are either determined automatically from previously expressed preferences (such as the amount of heat needed to achieve a desired room temperature) or are entered by the user (such as the time window within which the dishwasher must run). The biased randomisation arising from this probabilistic response ensures there is no undesirable peaking of demand.

The effect of this is to allow the supplier to obtain any shape of aggregate demand that is within the flexibility admitted by consumer needs, by choice of an appropriate signal. The supplier can minimise their balancing market and supply costs by setting the shape of $\boldsymbol{S}$ to induce demand that matches their generation contracts. A critical benefit of this method is that it is completely scalable - the same signal is sent to all consumers. The desired shape of demand arises as the aggregate of the diverse individual responses. The tariff offered to 
consumers can be fixed or have a time-of-day form that is consistent with the signal. If a time-of-day tariff is employed it simply acts to encourage consumers to choose suitable times to run appliances that are under wholly manual control. The trial described in the following sections provides a real-life test of this concept. It also tests the usability and benefits of a complex tariff scheme (further detailed in Section 3.3) combining a time-of-day tariff with an additional discount dependent on the availability of local photovoltaic generation. This overall concept, combining a non-tariff signalling scheme to manage randomised automated demand response with a complex tariff intended to influence demand response by human agency, has not been trialled previously. Results should be of wide interest given the critical need for stable and predictable demand response to realise the smart grid [1], [12].

\subsection{Community energy policy}

In 2014 the Department of Energy and Climate Change (DECC) published its first Community Energy Strategy [27] aimed at establishing a policy framework that would encourage the formation and sustainability of energy saving and generating projects initiated by communities and local government. The report recognised that to enable communities to benefit from the cost-saving potential of local electricity generation, both regulatory and technical innovation are required. In particular, "smart grid" technology is needed to enable local electricity consumption to be matched to generation. The strategy included a programme of pilot projects to stimulate this innovation under the rubric "Localised Energy Systems". The present trial, entitled Community Electricity Generation, Aggregation, and Demand Shaping (CEGADS) is one of these pilot projects.

\section{Storage heater control system}

\subsection{Demand shaping implementation}




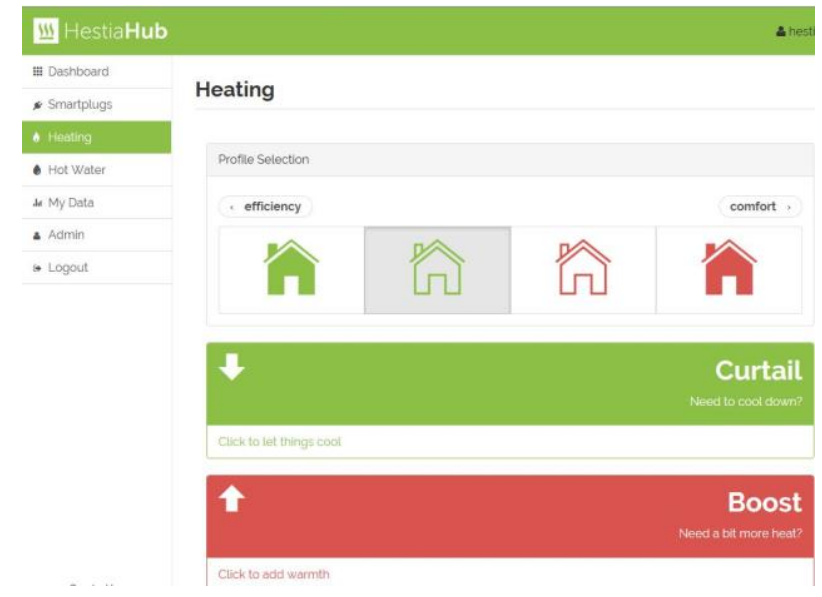

Fig.3 User interface for space heating

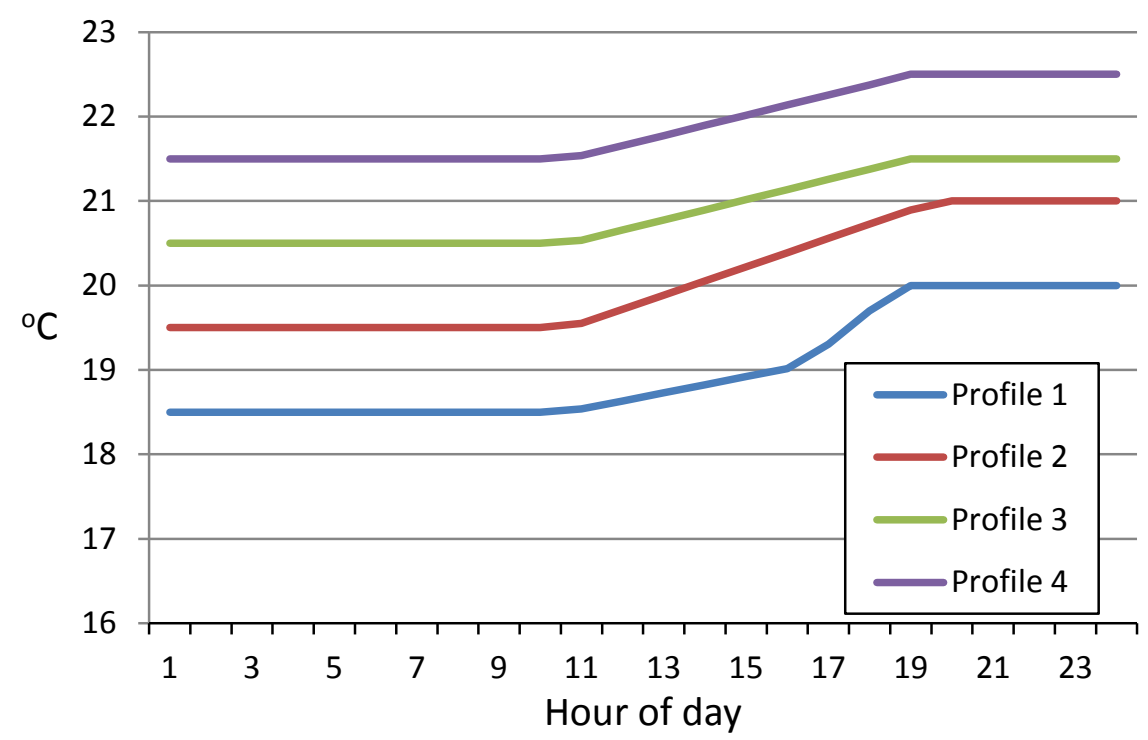

Fig. 4 Room temperature set point profiles

The in-home storage heating control system implemented for this trial (branded as a Hestia $\mathrm{Hub}^{2}$ ) achieves the reconciliation of user needs with the signalling of grid preferences in the following way. The user interface (shown in Figure 3) allows selection of one of four space heating temperature profiles indicated by a house icon. Each is associated with a nominal room temperature set point profile shown in Figure 4. These profiles are configured to be consistent with the range of actual temperatures found in UK homes [28], but they are not delivered by thermostatic control as there is no means of automatically adjusting the

\footnotetext{
${ }^{2}$ This device was designed by Exergy Devices Ltd. One of the authors (Boait) is a director and shareholder of this company.
} 
output control of a storage heater. They are in effect target temperatures which are used on an open loop basis for charging the heaters. Shortly before midnight each day the control system obtains a local weather forecast via the internet from the UK Meteorological Office using their DataPoint service [29]. The control system uses this forecast in two ways. Firstly, to make some adjustments to the target set point temperature profile, raising temperatures slightly if rain is forecast to reflect the chilling effect of higher humidity, and reducing them if sunshine is forecast to take advantage of solar gain, using a simple linear adjustment of the form:

$$
T_{a s}=T_{p}+k_{p} P-k_{g} U
$$

where $T_{a s}$ is the adjusted set point at a given time of day, $T_{p}$ is the profiled value at that time, $P$ is the forecast probability of precipitation, $U$ is the forecast ultraviolet index ${ }^{3}$, $k_{p}$ and $k_{g}$ are constants set so that maximum adjustment is $+/-1{ }^{\circ} \mathrm{C}$. Secondly, it uses the forecast external ambient temperature $T_{e}$ in conjunction with the weather-adjusted room temperature set point $T_{a s}$ and a value for the specific heat loss rate $L$ (in $\mathrm{kW} / \mathrm{K}$ ) of the dwelling to compute the expected heat load $Q_{i}$ in $\mathrm{kWh}$ for each $i$ th half-hour of the day:

$$
Q_{i}=L\left(T_{a s}-T_{e}\right) / 2
$$

An estimate of $L$ is provided to the control unit as a configuration parameter, then it is adjusted during operation by a learning algorithm to reflect the actual heat loss of each dwelling (determined from measured room temperatures, energy consumption, and the external temperature forecast) and the averaged heating effect of other electrical appliances. The required energy $Q_{i}$ must be available as heat not later than the $i$ th half-hour timeslot, but it can be supplied earlier as electricity charging a storage heater as long there is available capacity. The scheduling algorithm chooses a charging timeslot for $Q_{i}$ randomly from those

\footnotetext{
${ }^{3}$ Ultraviolet index is published in DataPoint to indicate the risk of sunburn but has proven to be a satisfactory proxy for insolation.
} 
that are possible, but with a bias to the random selection such that the probability $P_{j}$ of the $j$ th timeslot being selected is given by:

$$
P_{j}=A_{j} / \sum\left(A_{1} \ldots A_{i}\right)
$$

where $A_{1}$ to $A_{48}$ are attractiveness values obtained by inverting the supplier's signal $S$ using $A_{i}=S_{m a x}-S_{i}$. This scheduling process is repeated for all $Q_{i}$ with the assumption that at the start of the day (00:00) the heaters have no charge. The state of charge of the storage heaters is tracked during schedule creation using an empirical model for the output heat power $O_{i}$ :

$$
O_{i}=V_{i} F_{i-1} I
$$

where $V_{i}$ is the output control vent setting $(0.1=$ minimum, $1=$ fully open $), F_{i}$ is the charge fraction $(0=$ empty, $1=$ fully charged $)$ at the end of the $i$ th timeslot, and $I$ is the manufacturer's nominal input electrical power rating, used as a proxy for the maximum output heat power which is not given in specifications. The assumed output control settings are 0.1 from 00:00 to 07:00, then 0.4 to 17:00, then 1 for the rest of the day. For a set of storage heaters with total capacity $C \mathrm{kWh}$ the charge fraction when $Q_{i}$ is stored in the $j$ th halfhour timeslot is given by:

$$
F_{j}=F_{j-1}+\left(Q_{i}-O_{i} / 2\right) / C
$$

Values for $I$ and $C$ are provided to the control unit as configuration data. When full capacity is reached in a timeslot that timeslot is removed from the possible timeslots considered in the next iteration of equations (2) and (3). The final outcome of this scheduling process is a charging plan for the next day determining when the input to the storage heaters is switched on and off. Figure 5 shows the execution of a typical plan for storage heaters. 


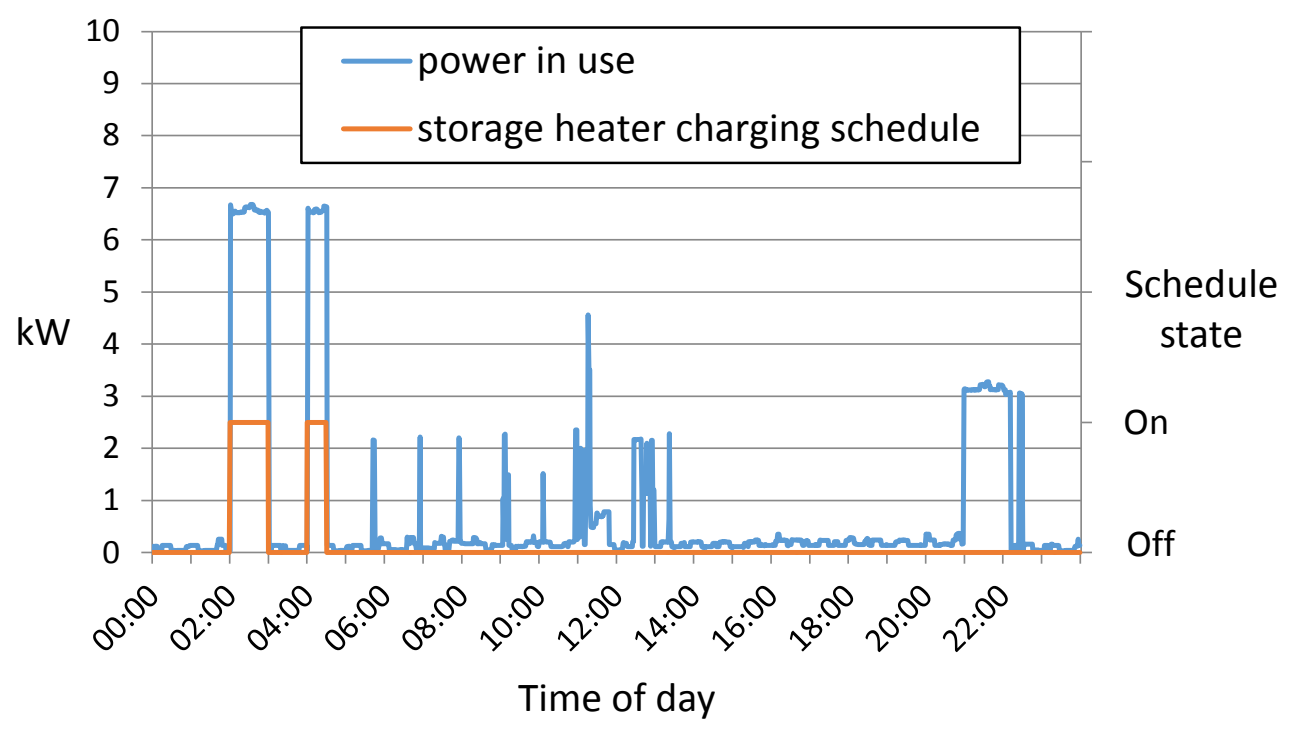

Fig. 5 Storage heater charging schedule and resulting electrical load

Dwellings with storage heating also usually have domestic hot water (DHW) provided from an insulated tank of 100-2001 capacity heated by an electric immersion heater. Conventionally the tank is heated overnight during the Economy 7 period using a simple timer so that most of the DHW demand is supplied using low cost electricity. The present control system makes use of this valuable energy storage resource for demand response by varying the timing of water heating during the day depending on the same grid supply attractiveness values that are employed for space heating in equation (3). Heating of the tank contents to $60^{\circ} \mathrm{C}$ is scheduled to commence in a timeslot that is randomly selected such that the probability $P_{j}$ of the $j$ th timeslot being selected is given by:

$$
P_{j}=A j / \sum\left(A_{1} \ldots A_{48}\right)
$$

Scheduled water heating can be seen in Figure 5 starting at 21:00. Since this time may not correspond closely to DHW usage, a function is included that detects when the tank has cooled to below $45^{\circ} \mathrm{C}$ and reheats it to $50^{\circ} \mathrm{C}$. This ensures that consumers always have hot water available but a substantial proportion of the energy needed is subject to demand response. 


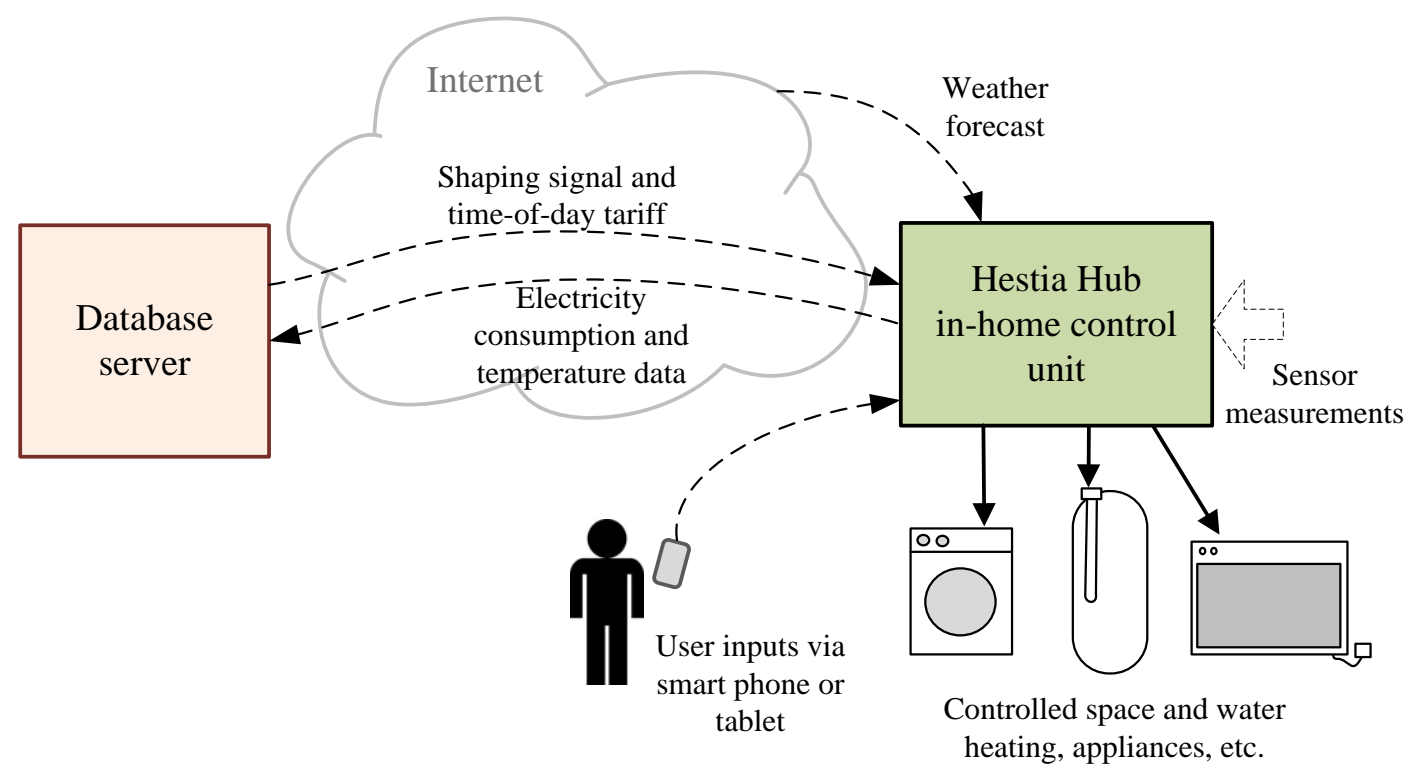

Fig.6 System diagram

A block diagram of the complete trial system is shown in Figure 6. The central database collects half-hour metering data and also a room temperature measurement. It publishes the signal $S$ to the in-home units and also the time-of-day tariff offered to the users. The control unit executes the algorithms described above and switches power to the storage heaters and immersion heater accordingly. It provides conventional "smart meter" functionality such as a display of current consumption and also allows the user to schedule operation of any appliance that can be supplied with electricity via a radio-controlled "smart plug" that switches on and off by command from the control unit. The user enters a time window within which the appliance must run, and the required running time. The control unit then schedules operation within the time window using the same biased randomisation process as described for the heating appliances. In the trial it is typically used for wet appliances that can be controlled simply by turning on and off at the mains and charging of battery-powered devices such as garden strimmers. 


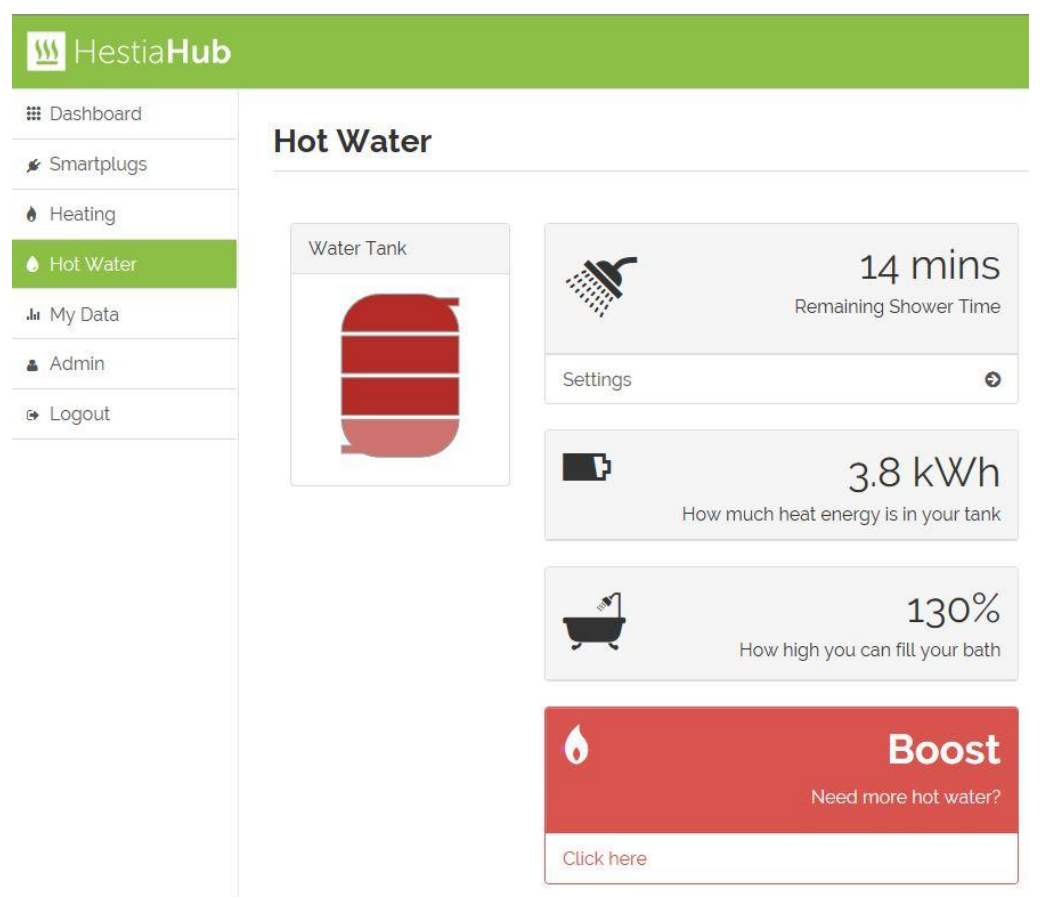

Fig. 7 User interface for hot water

The user interface is presented by a web server in the control unit that is accessible from within the home via a smart phone, tablet, or computer. Figures 3 and 7 show the user interface pages for space and water heating respectively. For space heating the user selects a room temperature profile as described above and also has the ability to initiate or cease heating immediately using the boost or curtail buttons. For hot water the graphic and icons indicate the volume of hot water available within the tank. The boost button initiates reheating of the hot water tank if exceptionally required.

\subsection{Trial objectives and participants}

The CEGADS trial has the objective of using a combination of demand response, a realistic time-of-day tariff provided by a retail electricity supplier, and community engagement to influence the aggregate electricity demand of the participants so that it makes efficient use of electricity generated by roof-mounted photovoltaic panels on some of the participant dwellings. In total 48 households have been recruited to take part. Of these, six have storage heating and it is results from these participants that are the main focus here. The 
homes with storage heating are single bedroom apartments arranged in two-storey buildings shown in Figure 8.

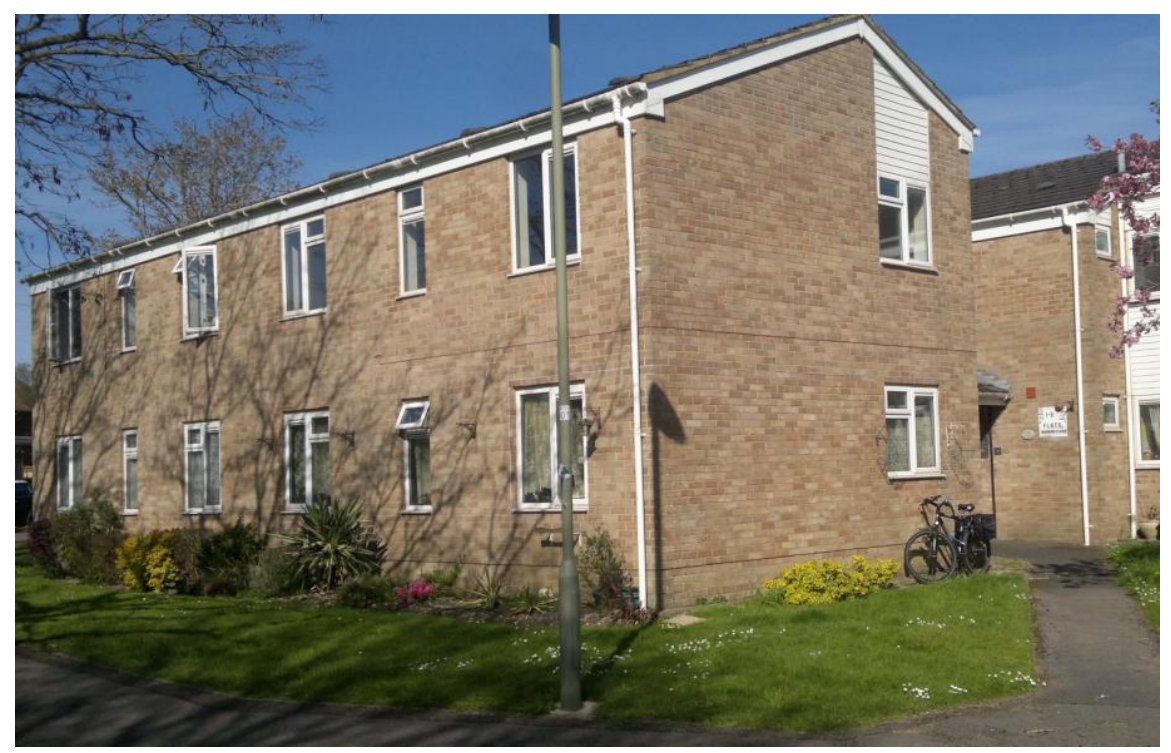

Fig. 8. Trial apartments with storage heating

A total of $52 \mathrm{kWp}$ of photovoltaic generating capacity is installed on 14 buildings within the trial. To enable participants in the trial to retain their existing electricity supplier and tariffs, the time-of-day tariff has been implemented as an incentive scheme where the difference between the actual cost of electricity to participants and the cost they would have incurred under the trial tariff is given to them in the form of credit vouchers exchangeable for goods at a supermarket chain associated with the supplier. The time-of-day tariff profile is shown in Figure 9 with an example of the demand shaping signal $S$ illustrating the alignment between the two. The effect of the signal values of 1 between 17:00 and 21:00 is to set the probability values from equation (3) to zero so that no appliance under control is scheduled to run during the peak tariff time. In addition to the time-of-day tariff, any exported electricity from the dwellings with PV that could be considered consumed by other participants (because their demand occurred at a time when PV export was available) was charged at 
$£ 0.065 / \mathrm{kWh}$. This created an incentive for all participants to use appliances in the middle of the day on sunny days.

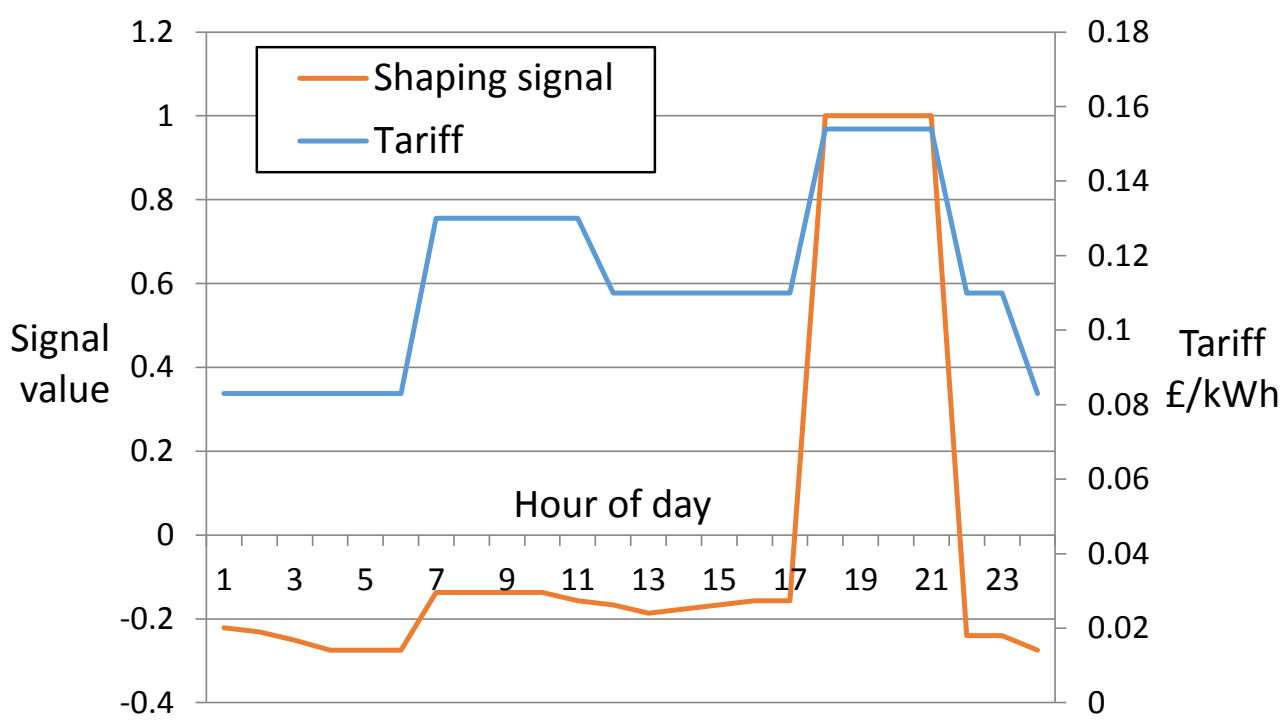

Fig. 9. Shaping signal and time-of-day tariff

\section{4. $\quad$ Results}

Results are presented to show the outcome against the two main goals outlined in Section 2:

- to exercise flexible control over the timing of electricity use for space and water heating taking account of the availability of local renewable generation;

- to provide automatic weather-dependent adjustment of storage heater charging and a convenient user interface.

\subsection{Electricity consumption}

Figures 10A - 10E show the average daily profiles of controlled electricity consumption by the user group for each of the months January, February and June 2016 with, as a comparator, the equivalent consumption profiles that would have occurred had these 
dwellings remained under conventional "Economy 7" off-peak control. These comparator profiles have been simulated with the assumption that the users would have successfully set their storage heaters manually to match the expected heating demand the following day - that is, a best case assumption that is not always realised. All the profiles are limited to electricity use that is subject to demand management. The dramatic reduction in peak demand from the six homes compared to that which would have occurred under conventional off-peak control can be seen by comparing the y-axis kWh scales for actual and simulated results.

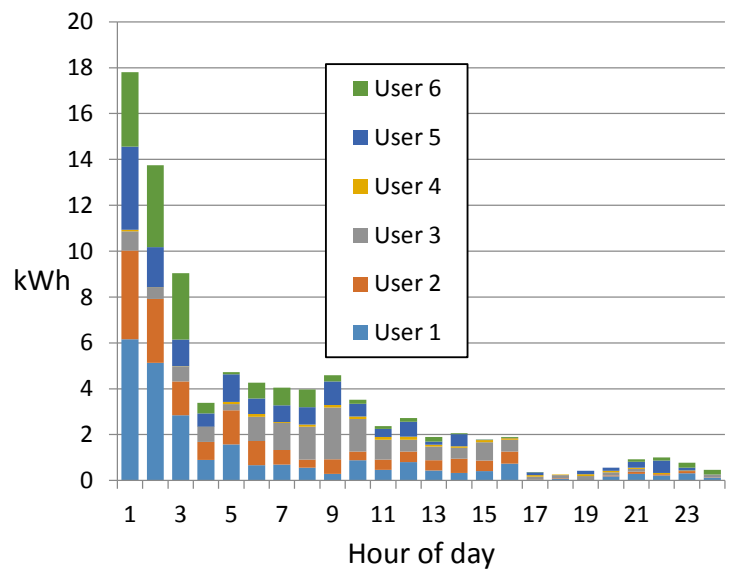

Fig. 10A. Actual average profile of "smart" controlled consumption in January

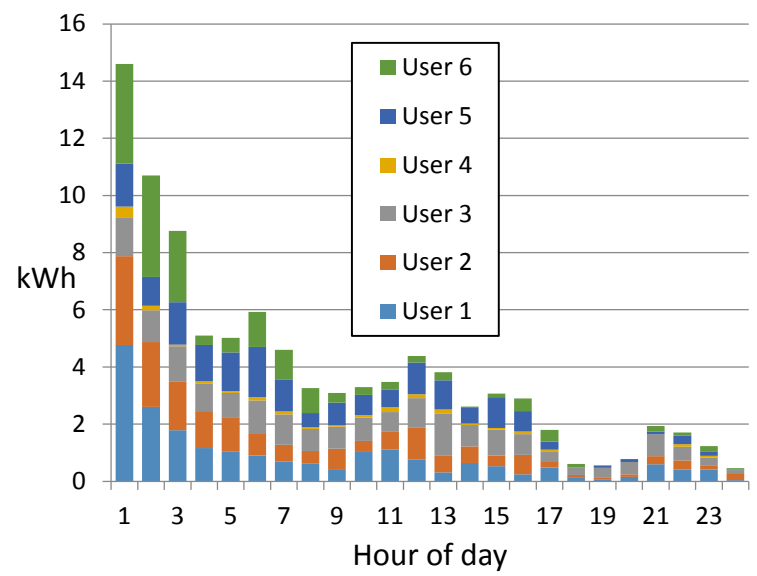

Fig. 10C. Actual average profile of "smart" controlled consumption in February

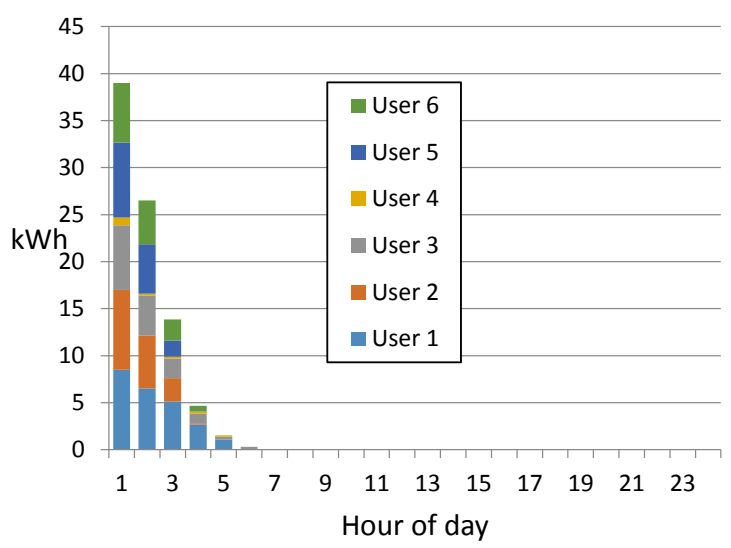

Fig. 10B. Simulated equivalent consumption in January using conventional "off-peak" control

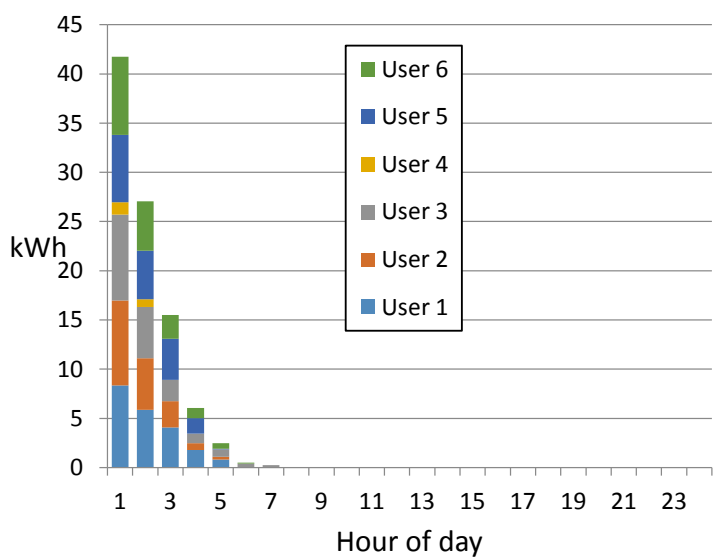

Fig. 10D. Simulated equivalent consumption in February using conventional "off-peak" control 


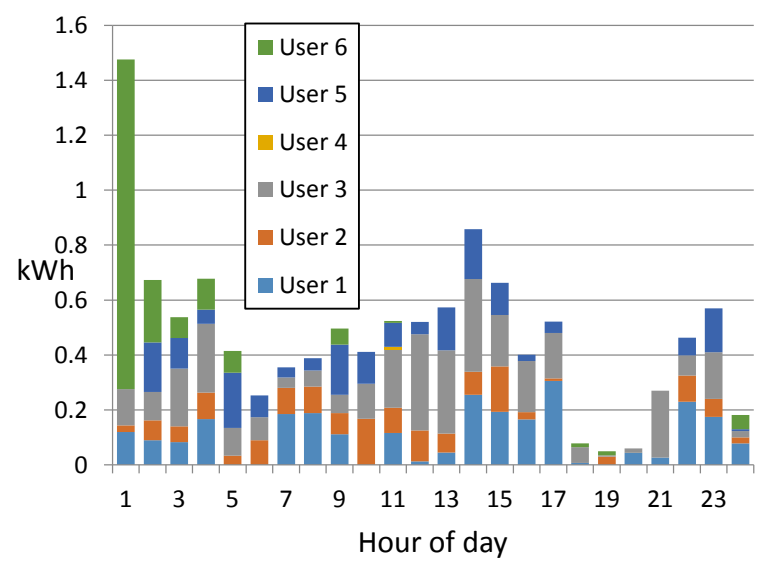

Fig. 10E. Actual average profile of "smart" controlled consumption in June

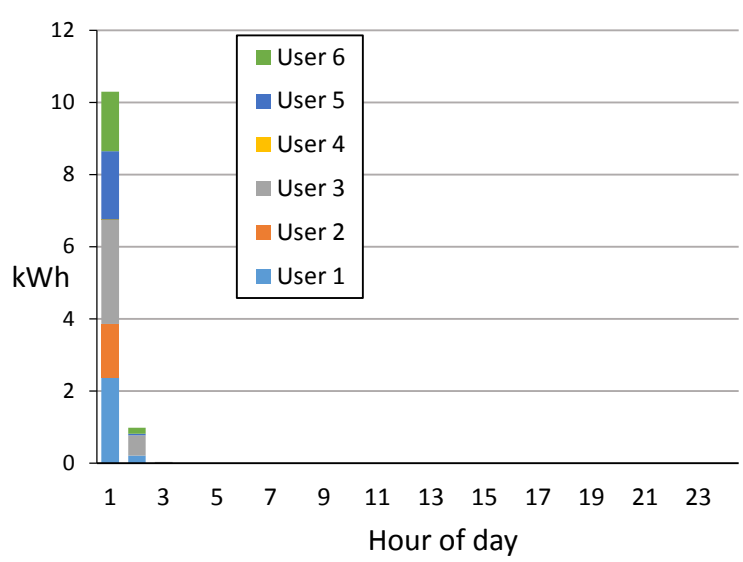

Fig. 10F. Simulated equivalent consumption in June using conventional "off-peak" control

Because the users are actually paying a conventional 'Economy 7' tariff (typically $£ 0.07 / \mathrm{kWh}$ during the 7 hour overnight period and $£ 0.16 / \mathrm{kWh}$ at other times) the demand shaping performed had to be quite cautious to avoid users experiencing high bills that would be distressing to receive and hence threaten their willingness to continue with the trial (although of course assurances of compensation were provided). The objectives of the shaping performed were therefore:

1. to minimise demand during the 17:00-21:00 peak tariff period;

2. to initially allocate about $33 \%$ of heating load outside the 7 hour overnight period in January, then increase this proportion as the year progressed, particularly in the hours when PV generation is available so that heating demand is satisfied by the local generation resource;

3. to spread demand within the 7 hour overnight period, to show how this technique can reduce the local co-incidence of demand from similar heating appliances.

The flexibility of demand was constrained by two factors. Firstly it is inherent in operation of a storage heater that it must be charged to deliver a useful heat output. So there is an unavoidable bias in demand towards the early part of a 24-hour cycle so that heat is available 
to users on waking and during the day. This effect is reinforced by the need to avoid the evening peak tariff period. Secondly, although the main heating cycle for hot water can be placed anytime during the day, the top-up function described in 3.1 tends to be invoked following a substantial hot water draw-off event. The small amounts of electricity use during the evening peak tariff period visible in Figures 10A, C and E are attributable to this cause, as are the higher levels of demand around 08:00-10:00. Table 1 quantifies the degree of success in meeting the demand shaping objectives during January - June 2016 using metrics linked to the three objectives.

Table 1. Demand shaping outcomes

\begin{tabular}{|l|l|l|l|l|l|l|}
\hline Objective no. and metric & January & Feb & March & April & May & June \\
\hline $\begin{array}{l}\text { 1.Heating demand during evening high } \\
\text { tariff period as \% of total heating } \\
\text { demand in month }\end{array}$ & 1.8 & 4.0 & 4.1 & 6.3 & 5.8 & 6.2 \\
\hline $\begin{array}{l}\text { 2. Daytime allocation of heating } \\
\text { demand excluding high tariff period \% }\end{array}$ & 32 & 38 & 37 & 40 & 48 & 55 \\
\hline $\begin{array}{l}\text { 2.Proportion of heating demand when } \\
\text { PV generated electricity available \% }\end{array}$ & 15 & 25 & 33 & 37 & 41 & 46 \\
\hline $\begin{array}{l}\text { 2. Proportion of heating demand when } \\
\text { PV generation available actually } \\
\text { satisfied by PV\% }\end{array}$ & 5 & 23 & 43 & 66 & 100 & 100 \\
\hline $\begin{array}{l}\text { 3. Reduction in peak heating demand } \\
\text { relative to Economy 7\% }\end{array}$ & 54 & 65 & 70 & 83 & 87 & 86 \\
\hline
\end{tabular}

The effectiveness of this system at adjusting the energy stored each day to match the weather-dependent actual heat load is illustrated by Figure 11. This shows the day-by-day correlation between total electricity consumed and local degree-days for the month of January 2016, for User 2. Total consumption rather than electricity supplied to the storage heaters is 
plotted because the Hestia control unit software takes account of all electricity consumed when calculating the thermal loss rate of the dwelling, and allows for typical appliance and lighting use when scheduling the storage heaters. This is an essential function for these relatively small and well-insulated dwellings as without it the storage heaters would be overcharged. However this means that the correlation is affected by day-to-day variations in appliance use.

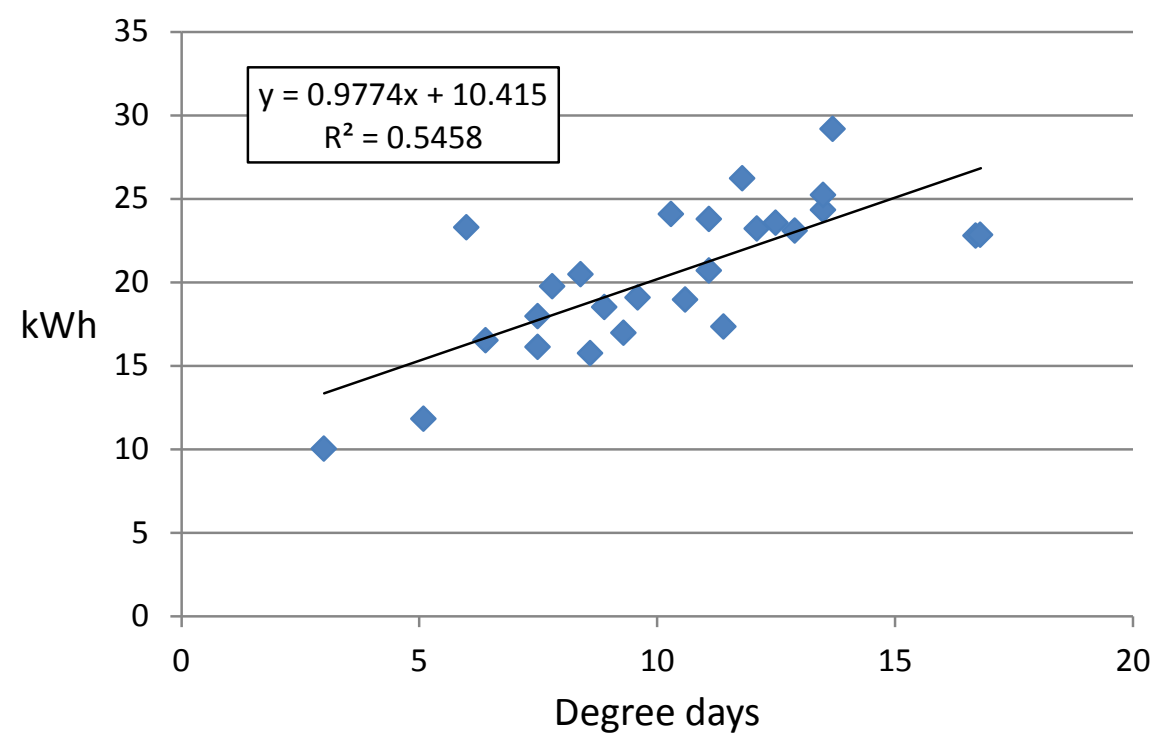

Fig. 11 Correlation between electricity consumed and degree days for User 2

Four of the users in the group (Users 1,2,5,6) displayed similar correlation, with $R^{2}$ values ranging from 0.42 to 0.6 for the same month. However, two users ( 3 and 4 ) show much lower correlation, one at 0.27 and the other effectively zero. The data collected by the control unit allows the extent of user intervention to be assessed, and it is clear that the two users with low correlation exercise much more "hands-on" control either through the web interface or by switching off the storage heaters and/or using auxiliary electric heating. In the case of User 4 this behaviour is associated with very low total energy consumption as can be seen in Figure 10, and a low measured room temperature. Figure 12 shows the daily average 
room temperatures measured for the user group in January showing consistent temperatures for 4 of the 6 and lower values for the other 2. This arose because the sensor was located in the hallway of each dwelling, and Users 4 and 6 chose to close internal doors and not heat the hallway.

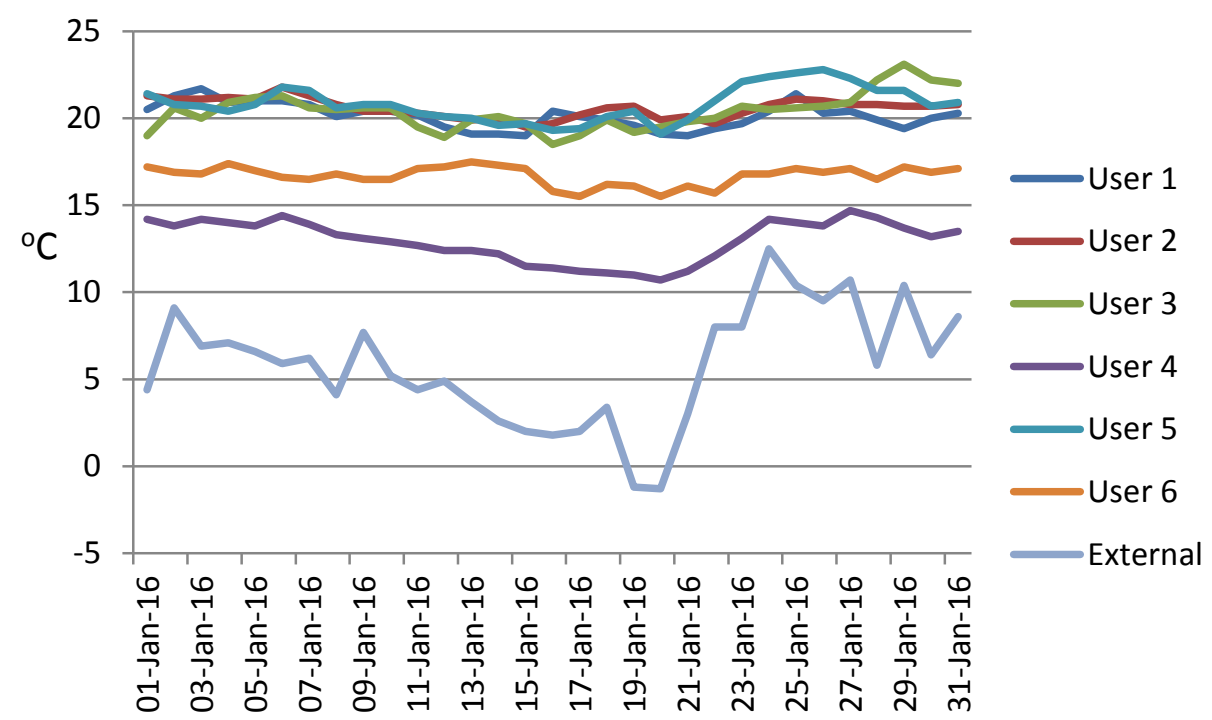

Fig. 12. Daily average internal temperature of the trial apartments and external temperature in January 2016

It was hoped that this automatic control of heater charging would provide energy savings for at least some users and would not increase consumption for others. This was assessed for the six users based on their billing data for the year prior to the start of the trial. Table 2 summarises the results. The consumption during the trial is an estimate correcting the measured data for differences in the degree-days of heating demand over the two heating seasons and including a prediction of each user's consumption during August and September 2016. Both adjustments use the applicable regression equation similar to that illustrated in Figure 11 but using monthly data which gives stronger correlation for all users $\left(R^{2}\right.$ values between 0.4 and 0.92 ). The table also shows the expected contribution of local PV-generated 
electricity to each user's total consumption for the trial year, calculated by doubling the amount actually consumed during January-June 2016.

Table 2. Weather-adjusted estimates of annual electricity consumption before and after intervention, with expected PV contributions

\begin{tabular}{|l|l|l|l|l|l|l|}
\hline & User 1 & User 2 & User 3 & User 4 & User 5 & User 6 \\
\hline Year prior to trial kWh & 6583 & 6205 & 3731 & 2383 & 4345 & 5362 \\
\hline Year of trial adjusted kWh & 6131 & 3919 & 4201 & 2360 & 4023 & 4849 \\
\hline Indicated reduction/rise \% & $-6.9 \%$ & $-37 \%$ & $13 \%$ & $-0.9 \%$ & $-7.4 \%$ & $-10 \%$ \\
\hline $\begin{array}{l}\text { Local PV generation } \\
\text { consumed in year of trial kWh }\end{array}$ & 630 & 370 & 488 & 356 & 442 & 390 \\
\hline
\end{tabular}

The impact on the users' costs of electricity from demand shaping and the project's tariff model is summarised in Table 3. The calculation method for this comparison was as follows:

- The estimated consumption for the trial year from Table 2 was costed using the Figure 9 tariffs and the tariff for local PV consumption of $£ 0.065 / \mathrm{kWh}$.

- An Economy 7 based counterfactual cost was estimated for the trial year assuming that each user continued to consume the same proportion of overnight electricity as before the trial and that the reduction or rise in consumption indicated in Table 2 did not occur.

- A common Economy 7 tariff for all users was assumed of $£ 0.0735$ for the overnight 7 hour period and $£ 0.16$ for other times, with a standing charge of $£ 0.157 /$ day. This tariff was actually being paid by 3 of the users who had the same supplier.

- The standing charge of $£ 0.157 /$ day was also applied to the project tariffs. 
Table 3. Estimated annual electricity cost with and without intervention

\begin{tabular}{|l|l|l|l|l|l|l|}
\hline & User 1 & User 2 & User 3 & User 4 & User 5 & User 6 \\
\hline Cost at project tariffs & $£ 634$ & $£ 435$ & $£ 563$ & $£ 308$ & $£ 493$ & $£ 5536$ \\
\hline Cost using Economy 7 & $£ 627$ & $£ 583$ & $£ 606$ & $£ 372$ & $£ 559$ & $£ 548$ \\
\hline Indicated cost reduction/rise \% & $+1 \%$ & $-25 \%$ & $-7 \%$ & $-17 \%$ & $-12 \%$ & $-2 \%$ \\
\hline
\end{tabular}

\subsection{User experience}

Semi-structured interviews were conducted by telephone with five out of the six trial participants who had electric storage heaters. The interviews were conducted between one and three weeks after installation of the Hestia Hub equipment, thus were in the early stages of the trial. The responses below illustrate the participants' overall attitude to the trial and to their electricity use, followed by their reaction to the Hestia device.

Trial participation. There were a variety of motivations for participating in the trial. One participant who had prior interest in energy saving said that the information and participation in the trial was very interesting: 'I' $m$ very very interested and I'm behind the trial. Very interested in the power that can be obtained by these natural resources' (User 1). Another participant was also interested in the potential benefits: 'I know it's a trial system, if and when it could be used, so we could be using local energy, then in due course that could be a good advantage. I don't know if that will ever come about' (User 6). One participant, who was not interested in the trial or reasons behind it, also appeared to be satisfied with participation: 'as far as I'm concerned, it does what it says on the tin. They promised me no inconvenience and that's what I've got' (User 4). 
Flexibility of consumption. When asked if they would be able to reduce electricity consumption at peak times the next day, three out of five participants mentioned that they would be able to do this easily or somewhat easily. Examples given were that they may be out during the afternoon, or change cooking habits, for example: 'I don't very often use the cooker anyway, it's usually a sandwich for me. ... But when you're living on your own, it's not worth really putting the cooker on just for one person. I might put the stove top on and heat up a tin of soup or something like that, but then I can usually put that in the microwave, it's quicker in there than anywhere else' (User 3)

Two participants mentioned that they wouldn't feel able to be flexible with electricity use in the evening, for example: 'Well, not the way I live at the moment ... the main meal is in the evening if I'm home, so that would be difficult. There's nothing much else ... so there's not much else I could reduce really' (User 6).

Demand reduction. All five respondents said that they had limited potential to reduce electricity demand. Of the three who mentioned there wasn't much they could do, three considered they were already at their minimum electricity usage, such as 'There's not a lot I could do really, because I'm all electric so I haven't any gas, I've no alternative.... I don't think I could reduce apart from the storage heaters, they could be turned down. I haven't had this one in the sitting room up to its maximum at all. When I last talked to Southern Electric, they said that the biggest part of your bill is the economy 7 part' (User 6). The other two mentioned small behavioural changes, such as 'Might not use the cooker. I definitely don't use the TV during the day. Probably in the weekend maybe for an hour or so' (User 3); and 'I believe I'm already at the minimum [consumption]' (User 4).

Understanding the Hestia equipment. Two participants said that they understood a little what the equipment was doing (User 1, User 2); and one mentioned that they 
understood, but would like more explanation: 'the graph ... when you look at the data, there's a graph on there, I don't understand that as much ... but probably when someone comes to see me they can explain more about it. ...all in the booklet that was left with me, which I read.' (User 3)

Understanding the display. One participant looked at the display several times a day 'I've had it now for it must be a week now, I check it every day, it's fascinating, I've never had the opportunity to see usage.' (User 1); while three other participants look at it once a day (User 2, User 3, User 6): 'I check it daily, just to see what I'm using at that time.' (User 6).

Controlling heating. There were mixed experiences of understanding the display and control interface. One participant understood and was using it well, for example: 'Oh yes you can put the heating up, yes I've used it yes, I'm using it through the tablet for the living room. The living room I've had to put up ... it was very cold Sunday night' (User 2). Another participant had problems understanding the interface: 'They provided me with a tablet, and I cannot use it. I am not computer literate' (User 4).

\section{Discussion}

The results indicate that this system for managing thermal storage has broadly met its objectives. The shaping of demand is very effective in lowering the peak demand from electric heating, so would free up headroom in distribution network capacity for growth in electric heating and electric car charging. An increasing proportion of heating demand has been diverted to the middle of the day where it was partly or wholly supplied by PV generation. However it was found that because of the need to charge a storage heater before the heat it holds is delivered there is a limit to the proportion of space heating energy that can be supplied within the mid-day low tariff period that also corresponds to the time when useful 
PV generation is available. In the case of water heating it is evident that the automatic mechanism that ensured some hot water was always available caused some demand to occur during the early evening tariff peak. In contrast under 'Economy 7' no automatically controlled demand can be placed outside the 7 hour overnight interval.

The marked differences in energy consumption between users, despite all being single occupants of nearly identical dwellings, give a strong indication of the interaction between energy use behaviour and the impact of this technology. Users 1,2 and 5 made relatively little use of the user interface so the control unit determined the amount of energy delivered to both the storage heaters and hot water tank. User 6 intervened to manage hot water heating manually, as can be seen in hour 1 of Fig. 9E, but left the control unit to manage the storage heaters. For users 1, 5, and 6 this results in a modest energy saving over a year, whereas for User 2 the saving is substantial, probably because prior to intervention they did not use the manual storage heater input control effectively. User 4 made frequent use of the user interface to control heating, while User 3 intervened to turn storage heating off at the wall switch preventing the control unit from having any effect. These two had the lowest energy use prior to intervention, but the control unit was unable to deliver energy savings that were additional to the users' own efforts and for User 3 caused an increase in consumption. This outcome is consistent with wider research concerning heating controls. For example a DECC study [30] identified five behavioural archetypes including "rationers" who manually control heating to minimise cost and "hands-off" who leave controls alone if they are delivering adequate comfort. Users 3 and 4 correspond to the former group and 1, 2 and 5 the latter.

The cost comparison in Table 3, showing useful potential savings for four out of six users and no significant impact for the other two, suggests that the business model represented by the project tariffs combined with the demand shaping technology can produce benign outcomes for a range of user behaviours and consumption patterns. The cost saving 
shown for User 3 despite an increase in consumption arises because their usage matches the project tariffs better than 'Economy 7', as does that of User 4. Had the users actually been paying the project tariff it would have been possible to optimise the control signal more closely to that tariff and thereby deliver further cost savings, whereas under the trial the signal was a compromise that mitigated the increase in their incurred costs resulting from movement of demand out of the low tariff 7-hour overnight period. This provides some confirmation of the conclusion in [31] that fuel-poor households could benefit from time-ofuse and real time tariffs, given supportive technology that allows them to place demand when surplus renewable energy is available and avoid high cost peak demand periods. In the longer term, the more efficient use of the distribution network demonstrated by this system should give rise to the considerable "smart grid" savings identified in [1].

With effective regulation these savings, which could not be reflected in the project tariffs, should be passed on to the consumers who generate them through their acceptance and use of demand response technology combined with more complex tariffs. However it is important to note that the users in this project have been assisted by intensive engagement of the project team to explain and discuss the novel aspects of the concept and respond to the kind of concerns exemplified by the user quotations above. This confidence-building activity will be essential in any larger deployment.

To fully exploit the investment involved in a large scale deployment of this technology, further work is needed to develop its potential for contributing to the balancing services procured by the UK national system operator (National Grid). These services [32] require the provider to deliver a short term increase or decrease in electricity demand in response to a signal from the system operator, or in response to variations in the supply frequency. This trial has demonstrated the capability of the Hestia control units to reconcile user needs and preferences with those of the retail electricity supplier, so it is a logical and feasible step to 
include requests for balancing services in the reconciliation. The server system shown in Figure 6 could aggregate and record a valuable overall response from the subset of Hestia units, within a potentially large population, that would be able to act on a given service request without compromise to the user needs or comfort.

\section{Conclusions}

This trial has sought to show how legacy storage heating can be updated to provide a valuable service to its users and the community at large in the era of smart metering and the smart grid. This has required a fully integrated realisation of many of the features widely regarded as necessary in an electricity system that can cope with increasing renewable generation and increasing demand from electric heating and vehicles. These include:

- automated demand response that prioritises user needs;

- automated demand control that responds to signalling from the electricity supplier in a way that flattens the daily profile of demand and increases consumption of local generation;

- a complex tariff scheme combining static time-of-day and real-time pricing;

- an accessible and supportive user interface that combines smart meter functions with heating control to make efficient use of energy storage;

- an effective programme of user engagement.

The quantitative and qualitative results obtained show that this synthesis of smart grid elements has been broadly successful in achieving its aims for this small group of consumers. There are three critical lessons learned from the user experience that should be applied to any larger scale implementation. First, that it must be easily possible for users who prefer to manage their energy use in a hands-on way to continue to do so even if this means overriding 
the demand response. These are often very frugal users. Secondly, complex tariffs need not be unacceptably confusing if their exploitation is supported by the technology and they are reasonably intuitive. In this case users needed only to look out of the window to know when cheap PV electricity was available. The third, related, lesson has been that the demand response was achieved through a combination of carefully-designed technology, knowledge transfer and confidence-building, with skilled friendly people available to explain the new arrangements and respond to concerns.

\section{Acknowledgements}

The authors would like to thank the Engineering and Physical Sciences Research Council (EPSRC) and Innovate UK for providing the financial support for this study as part of the CEGADS project (EP/M507210/1). The valuable comments from reviewers, and the work by Westmill Sustainable Energy Trust to recruit and support participants for this trial, are also gratefully appreciated.

\section{References}

[1] National Infrastructure Commission, Smart Power (2016).

https://www.gov.uk/government/uploads/system/uploads/attachment_data/file/505218/IC_En ergy_Report_web.pdf

[2] D. Pudjianto, P. Djapic, M. Aunedi, C. K. Gan, G. Strbac, S. Huang, D. Infield, Smart control for minimizing distribution network reinforcement cost due to electrification, Energy Policy 52 (2013) 76-84.

[3] Ofgem, Making the electricity system more flexible and delivering the benefits for consumers (2015).

https://www.ofgem.gov.uk/sites/default/files/docs/2015/09/flexibility_position_paper_final_0 . $\mathrm{pdf}$ 
[4] Department of Communities and Local Government, English housing survey headline report 2014 to 2015 section 2 housing stock table 2.7 (2016).

https://www.gov.uk/government/statistics/english-housing-survey-2014-to-2015-headlinereport

[5] Department for Business, Energy, and Industrial Strategy, Fuel Poverty detailed tables (2014), Table 11a https://www.gov.uk/government/statistics/fuel-poverty-detailed-tables$\underline{2014}$

[6] D. J. C. MacKay, Sustainable Energy: Without the Hot Air, ISBN 9780954452933. UIT Cambridge (2009).

[7] British Standard Institute (BSI), Radio teleswitches for tariff and load control, BSI 7647. London: BSI (1993).

[8] Ofgem, The state of the market for customers with dynamically teleswitched meters (2013). https://www.ofgem.gov.uk/ofgem-publications/82288/state-market-customersdynamically-teleswitched-meters.pdf

[9] City of Westminster, Purchase of Communal Electricity and Gas 2014/15 (2014). http://www3.westminster.gov.uk/CSU/Cabinet\%20Member\%20Decisions/Housing,\%20Rege neration, \%20Business\%20and\%20Economic\%20Development\%202013-15/2013\%20\%202014/08\%20\%20Purchase $\% 20$ of $\% 20 \mathrm{Communal} \% 20$ Electricity\%20and\%20Gas\%20201415/Cabinet\%20Member\%20Report\%20\%20Purchase \%20of\%20Communal\%20Electricity\%20and\%20Gas.pdf

[10] Department of Energy and Climate Change, Smart meters: a guide (2013). https://www.gov.uk/guidance/smart-meters-how-they-work

[11] Department of Energy and Climate Change, The future of heating - meeting the challenge (2013). https://www.gov.uk/government/publications/the-future-of-heatingmeeting-the-challenge

[12] F. Teng, M. Aunedi, G. Strbac, Benefits of flexibility from smart electrified transport and heating in the future UK electricity system (2016). Applied Energy 167, 420-431. 
[13] S. J. Darby, Balancing the system comfortably? Electric storage heating and residential demand response (2016). Proc. of Behave 2016, $4^{\text {th }}$ European conference on behaviour and energy efficiency, Coimbra 8-9 Sept.

[14] M. Ali, A Safadarian, M. Lehtonen, Risk-constrained framework for residential storage space heating load management (2015). Electric Power Systems Research 119, 432-438.

[15] P. Tuohy et. al., Orchestration of renewable generation in low energy buildings and districts using energy storage and load shaping (2015). Energy Procedia 78, 2172-2177.

[16] Centre for Sustainable Energy, Nightmare storage heaters? (2015)

https://www.cse.org.uk/news/view/1765

[17] Peabody Housing Association, Storage heaters advice (2014)

https://www.youtube.com/watch?v=Xo2Fs5vSNqk

[18] Consumer Focus. From devotees to the disengaged. A summary of research into energy consumers' experiences of Time of Use tariffs and Consumer Focus's recommendations.

[19] Bouzarovski, S. and Petrova, S. A global perspective on domestic energy deprivation: Overcoming the energy poverty-fuel poverty binary. Energy Research and Social Science 10, (2015), 31-40

[20] Peffer, T., Pritoni, M., Meier, A. , Aragon, C. and Perry, D. (2011) How people use thermostats in homes: A review. Building and Environment 46 (12), (2011), 2529-2541

[21] Energy Technologies Institute, Smart Systems and Heat (2015)

http://www.eti.co.uk/smart-systems-and-heat-consumer-challenges-for-low-carbon-heat/

[22] A. Mohensian-Rad, A. Leon-Garcia, Optimal residential load control with price prediction in real-time electricity pricing environments, IEEE Trans. Smart Grid 1(2) (2010) 120-132.

[23] S. D. Ramchurn, P. Vytelingum, A. Rogers, N. R. Jennings, Agent-Based Control for Decentralised Demand Side Management in the Smart Grid, Proc. of 10th Int. Conf. on Autonomous Agents and Multiagent Systems (AAMAS 2011), Taipei, (2011) 5-12. 
[24] A. Roscoe, G. Ault, Supporting high penetrations of renewable generation via implementation of real-time electricity pricing and demand response, IET Renew. Power Gener. 4(4) (2010) 369-382.

[25] R. M. Rylatt, J. R. Snape, P. Allen, B. M. Ardestani, P. Boait, E. Boggasch, D. Fan, G. Fletcher, R. Gammon, M. Lemon, V. Pakka, C. Rynikiewicz, M. Savill, S. Smith, M. Strathern, L. Varga, Exploring Smart Grid Possibilities: A Complex Systems Modelling Approach, Smart Grid 1 (2015) 1-15.

[26] P. J. Boait, J. R. Snape, B. M. Ardestani, Accommodating renewable generation through an aggregator-focused method for inducing demand side response from electricity consumers, IET Renewable Power Generation 7 (6) (2013) 689-699.

[27] Department of Energy and Climate Change, Community Energy Strategy Full Report (2014)

https://www.gov.uk/government/uploads/system/uploads/attachment_data/file/275163/20140 126Community_Energy_Strategy.pdf

[28] G. M.Huebner, M. McMichael, D. Shipworth, M. Shipworth, M. Durand-Daubin, A. Summerfield, The reality of English living rooms - A comparison of internal temperatures against common model assumptions, Energy and Buildings 66 (2013), 688-696

[29] Met Office, DataPoint (2016). http://www.metoffice.gov.uk/datapoint

[30] Department of Energy and Climate Change, What people want from their heating controls; a qualitative study (2014)

https://www.gov.uk/government/uploads/system/uploads/attachment_data/file/251785/what people_want_from_their_heating_controls_qualitative_study.pdf

[31] S.J. Darby, Metering: EU policy and implications for fuel poor households, Energy Policy 49 (2012), 98-106.

[32] National Grid, Keeping the Electricity Transmission System in balance (2016), http://www2.nationalgrid.com/uk/services/balancing-services/ 